\title{
PERFIL EPIDEMIOLÓGICO DA SÍFILIS CONGÊNITA NO BRASIL NO PERÍODO DE 2012 A 2018
}

\author{
EPIDEMIOLOGICAL PROFILE OF CONGENITAL SYPHILIS IN BRAZIL \\ FROM 2012 TO 2018
}

Cleisla Tamires Lacerda Silva ${ }^{1}$

Karla Pereira Vasconcelos ${ }^{2}$

RESUMO: OBJETIVO: avaliar a notificação da sífilis congênita no Brasil entre os anos de 2012 a 2018, de acordo com a base de dados do SINAN, descrevendo o perfil epidemiológico das gestantes cujos recém-nascidos apresentaram sífilis congênita, verificando a realização de pré-natal e do tratamento das gestantes e seus parceiros. MÉTODOS: estudo transversal, descritivo, com abordagem quantitativa, realizado por meio de coleta de dados na plataforma on-line SINAN. A população de estudo foi composta por todos os casos de sífilis congênita notificados no Brasil no período entre 2012 e 2018. RESULTADOS: foram notificados 134.409 casos de sífilis congênita no Brasil entre de 2012 a 2018. Em relação as variáveis, a faixa etária da maioria das mães estava entre 20 e 29 anos, a escolaridade mais observada foi da $5^{\circ}$ a $8^{\circ}$ série incompleta, a maioria realizou o pré-natal e foi diagnosticada com sífilis durante o mesmo, a maior parte das gestantes recebeu um tratamento inadequado, e mais da metade dos parceiros não foram tratados. CONCLUSÃO: Apesar de ser uma infecção que apresenta baixo custo e facilidade para sua prevenção, a sífilis congênita permanece como uma infecção de difícil controle no Brasil, gerando impactos na saúde materna infantil. Para mudar essa realidade, é necessário que o pré- natal do país tenha sua qualidade melhorada. É preciso que os profissionais se empenhem em acolher, identificar e orientar gestantes portadoras de sífilis congênita, e sensibilizá-las para realizarem um tratamento adequado para não fornecer riscos a seu bebê.

Palavras chave: Cuidado pré-natal. Epidemiologia. Perfil de Saúde. Sífilis. Sífilis Congênita.

\footnotetext{
1 Graduanda do Curso de Enfermagem da Universidade Estadual da Paraíba - UEPB, cleislalacerda@gmail.com.

${ }_{2}$ Graduanda do Curso de Enfermagem da Universidade Estadual da Paraíba - UEPB, karlapv2017@gmail.com.
} 
ABSTRACT: OBJECTIVE: to evaluate the notification of congenital syphilis in Brazil from 2012 to 2018, according to the SINAN database, describing the epidemiological profile of pregnant women whose newborns had congenital syphilis, verifying prenatal care and treatment of pregnant women and their partners. METHOD: Crosssectional, descriptive study with quantitative approach, performed through data collection on the SINAN online platform. The study population consisted of all cases of congenital syphilis reported in Brazil between 2012 and 2018. RESULTS: 134,408 cases of congenital syphilis in Brazil were reported from 2012 to 2018. Regarding the variables, the age range of most mothers were between 20 and 29 years old, the most frequently observed schooling was from 5th to 8th grade incomplete, most of them had prenatal care and were diagnosed with syphilis during it, most pregnant women received inadequate treatment, and more than half of the partners were not treated. CONCLUSION: Despite being a low cost and easily preventable infection, congenital syphilis remains an infection that is difficult to control in Brazil, causing impacts on maternal and child health. To change this reality, the country's prenatal care needs to be improved. Professionals must strive to accommodate, identify and guide pregnant women with congenital syphilis, and sensitize them to receive appropriate treatment to avoid risk to their baby.

Keywords: Prenatal Care. Epidemiology. Health profile. Syphilis. Congenital Syphilis. 


\section{INTRODUÇÃO}

A sífilis é uma infecção bacteriana, exclusiva do ser humano, causada pela espiroqueta Traponema pallidum, que foi descoberta em 1905. Pode ser transmitida por via sexual, transfusão de sangue e derivados, ou transmissão vertical quando a mãe transmite para o feto, esta chamada de sífilis congênita (BRASIL, 2015).

A sífilis congênita ainda é observada como um importante problema de saúde pública, mesmo se tratando de uma infecção de diagnóstico simples e completamente evitável por meio do tratamento da gestante e de seu parceiro (COSTA et al., 2013). Em 2012, a sífilis congênita sofreu mundialmente uma redução de 39\% em sua incidência. As regiões que apresentaram maior incidência foram a África e o Sudeste Asiático, mesmo este mostrando uma grande redução de casos. Os países das Américas também obtiveram uma diminuição do número de casos, porém o Brasil foi uma exceção, não apresentando redução (REIS et al, 2018).

A sífilis adquirida pode ser classificada conforme o tempo de infecção (recente ou tardia), ou de acordo com suas manifestações clínicas (primária secundária, latente eterciária). A sífilis primária permanece por duas a seis semanas e é caracterizada por úlcera indolor, única, de base endurecida e fundo limpo, chamada de cranco-duro, associada com a linfadenopatia inguinal. Na secundária é observado o aparecimento de erupções cutaneomucosas sintomáticas que surgem em seis semanas a seis meses após a infecção. Na latente não se observam sinais e sintomas, e a terciária ocorre após longo período de latência, de dois a quarenta anos após infecção, e apresenta-se com inflamação e destruição tecidual, podendo atingir o sistema nervoso e cardiovascular (BRASIL, 2015).

Já a sífilis congênita pode ser classificada como precoce ou tardia. A precoce pode se manifestar até o segundo ano de vida e mais de $50 \%$ das crianças são assintomáticas ao nascimento. Quando surgem, os sintomas são prematuridade, baixo peso, hepatomegalia, lesões de pele, periostite, sofrimento respiratório, 
icterícia, anemia, entre outros. (BRASIL, 2015). As crianças infectadas que não passa por identificação e tratamento adequados estão sujeitas a apresentarem manifestações da sífilis congênita tardia, que afetam o sistema nervoso central, ossos, articulações, pele, dente e olhos, entre elas está a tríade de Hutchinson, que engloba ceratite intersticial, surdez devido lesão no oitavo nervo craniano e os dentes de Hutchinson, tendo assim sérias consequências na sua vida adulta (COOPER et al., 2016).

A sífilis congênita pode acarretar serias consequências, a exemplo de abortamentos, natimorto, parto pré-termo e manifestações clínicas, porém só os casos muito graves são aparentes clinicamente no nascimento, e sua transmissão pode acontecer em qualquer fase da gestação ou estágio da doença na mãe, (BRASIL, 2019).

O estágio da doença na gestante, principalmente no primário e secundário, e o tempo de exposição no feto influenciam na infecção fetal. A taxa de transmissão vertical de sífilis da gestante para o feto é de cerca de $80 \%$ intraútero, porém também pode ocorrer a transmissão durante o parto vaginal, caso a mãe possua alguma lesão sifilítica. No feto, a infecção ocasiona de 30\% a 50\% de morte dentro do útero, parte pré-termo ou morte neonatal (BRASIL, 2019).

A sífilis congênita pode ser evitada quando é identificada e tratada adequadamente na gestante e no seu parceiro sexual (BRASIL, 2015). As gestantes diagnosticadas com sífilis devem receber um tratamento adequado com penicilina de acordo com seu estágio de infecção. Mulheres que já apresentaram alergia à penicilina necessitam passar por um processo de dessensibilizarão para serem tratadas com o medicamento (COOPER et al., 2016). As gestantes devem ser testadas durante o pré-natal na primeira consulta, início do terceiro trimestre, e na internação para o parto. Os recém-nascidos com mães diagnosticadas com sífilis gestacional, mesmo que estas tenham sido tratadas, devem realizar o teste não treponêmico periférico, não sendo coletado o sangue do cordão umbilical, pois este possui sangue materno (BRASIL, 2019). Um número baixo de casos de sífilis congênita pode indicar um programa eficiente de controle de transmissão vertical, mas também podem mostrar que há casos que não são notificados. Um número alto de casos aponta que ocorrem erros na assistência, sem intervenção satisfatória. 
Desta maneira, o número de casos notificados resulta da capacidade de intervenção do serviço para diagnosticar e tratar as gestantes e seus parceiro, diminuindo a transmissão, e também da capacidade de notificar os casos (DOMINGUES; LEAL, 2016).

Diante disso, o governo brasileiro criou a Rede Cegonha em 2011, que possui, entre outras ações, a prevenção e tratamento de DST, garantindo o financiamento do teste rápido de sífilis no pré-natal (BRASIL, 2011). Para isso, instituiu em 2012 a Portaria $n^{\circ} 77$, que dispõe sobre a realização de testes rápidos na atenção básica para detecção de sífilis e HIV (BRASIL, 2012).

Desta forma, faz-se necessário a investigação da real situação da sífilis congênita em nosso país e realização de pesquisas referentes ao tema. Assim, este estudo tem por objetivo avaliar a notificação da sífilis congênita no Brasil entre os anos de 2012 a 2018, de acordo com a base de dados do Sistema Nacional de Agravos e Notificações - SINAN, descrevendo o perfil epidemiológico das gestantes cujos recém-nascidos apresentaram sífilis congênita, verificando a realização de prénatal e do tratamento das gestantes e seus parceiros.

\section{METODOLOGIA}

Trata-se de um estudo transversal, descritivo, com abordagem quantitativa, realizado por meio de coleta de dados na plataforma on-line SINAN. Esta plataforma é alimentada essencialmente pela notificação e investigação de casos de doenças e agravos que fazem parte da lista nacional de doenças de notificação compulsória. A população de estudo foi composta por todos os casos de sífilis congênita notificados no Brasil no período entre 2012 e 2018 (134.409), não sendo utilizado os casos de 2019, pois foram divulgados os dados de casos notificados até 30/06/2019, desta forma não demonstrariam todos os casos registrados neste ano. A coleta de dados foi realizada em dezembro de 2019.

As variáveis investigadas foram o número de casos de sífilis congênita, idade e escolaridade das mães que tiveram bebês diagnosticados com sífilis congênita, 
realização do pré-natal, momento de diagnóstico materno e tratamento da mãe e seus parceiros. Os dados são apresentados através de tabelas, e expressos em frequências absolutas e relativas.

Por se tratar de um estudo que utiliza dados do SINAN, uma plataforma online do Ministério da Saúde na qual os dados estão disponíveis para livre acesso, o estudo não necessitou de aprovação do comitê de ética em pesquisas em seres humanos.

\section{RESULTADOS}

Verificou-se que de 2012 a 2018 foram notificados 134.409 casos de sífilis congênita no Brasil, e destes, 133.972 foram diagnosticados em crianças menores de um ano, havendo um aumento de casos notificados a cada ano (tabela 1).

Tabela 1 - Casos de sífilis congênita segundo idade da criança por ano de diagnóstico. Brasil, 2012 a 2018.

\begin{tabular}{|c|c|c|c|c|c|c|c|c|}
\hline Brasil & 2012 & 2013 & 2014 & 2015 & 2016 & 2017 & 2018 & Total \\
\hline $\begin{array}{l}\text { Idade da } \\
\text { criança }\end{array}$ & $n(\%)$ & $n(\%)$ & $n(\%)$ & $n(\%)$ & $n(\%)$ & $n(\%)$ & $n(\%)$ & $n(\%)$ \\
\hline $\begin{array}{l}\text { Menos } \\
\text { de } 7 \text { dias }\end{array}$ & $\begin{array}{l}11235 \\
(96,2)\end{array}$ & $\begin{array}{l}13499 \\
(96,4)\end{array}$ & $\begin{array}{l}15756 \\
(96,3)\end{array}$ & $\begin{array}{l}19003 \\
(96,4)\end{array}$ & $\begin{array}{l}20527 \\
(96,2)\end{array}$ & $\begin{array}{l}24191 \\
(96,7)\end{array}$ & $\begin{array}{l}25456 \\
(96,8)\end{array}$ & $\begin{array}{c}129667 \\
(96,4)\end{array}$ \\
\hline $\begin{array}{c}7 \text { a } 27 \\
\text { dias }\end{array}$ & $207(1,8)$ & $231(1,6)$ & $270(1,7)$ & $337(1,7)$ & 356 (1 & 375 & $433(1,6)$ & $\begin{array}{l}2209 \\
(1,7)\end{array}$ \\
\hline $\begin{array}{c}28 \text { a } 364 \\
\text { dias }\end{array}$ & 193 & $243(17)$ & 2 & 307 & . & & & $\begin{array}{r}2096 \\
(1,6)\end{array}$ \\
\hline 1 ano & 21( & 27 & 1 & & 37 & 34 & 27 & $199(0,1)$ \\
\hline $\begin{array}{l}2 \text { a } 4 \\
\text { anos }\end{array}$ & $12(0,1)$ & $5(0,0)$ & $18(0,1)$ & $28(0,1)$ & $28(0,1)$ & $21(0,1)$ & $18(0,1)$ & $130(0,1)$ \\
\hline $\begin{array}{c}5 \text { a } 12 \\
\text { anos }\end{array}$ & $11(0,1)$ & $4(0,0)$ & $7(0,0)$ & $9(0,0)$ & $12(0,1)$ & $15(0,1)$ & $25(0,1)$ & $83(0,1)$ \\
\hline Ignorado & - & 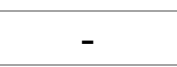 & te & 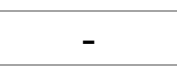 & - & & $18(0,1)$ & $24(0,0)$ \\
\hline Total & $\begin{array}{l}11679 \\
(100)\end{array}$ & $\begin{array}{l}14009 \\
(100)\end{array}$ & $\begin{array}{c}16354 \\
(100)\end{array}$ & $\begin{array}{l}19719 \\
(100)\end{array}$ & $\begin{array}{c}21331 \\
(100)\end{array}$ & $\begin{array}{c}25009 \\
(100)\end{array}$ & $\begin{array}{c}26307 \\
(100)\end{array}$ & $\begin{array}{c}134409 \\
(100)\end{array}$ \\
\hline
\end{tabular}

Fonte: MS/SVS/SINAN - Departamento de Doenças de Condições Crônicas e Infecções Sexualmente Transmissíveis. 
Em relação à idade das mães de crianças infectadas com sífilis congênita (tabela 2), as faixas etárias variaram de 10 a 40 anos ou mais, sendo que a maioria tinha entre 20 e 29 anos ( $n=70489 / 52,4 \%$ ). De 40 anos ou mais, foram registrados $2601(1,9 \%)$ casos. Destaca-se o número de gestantes com idade ignorada 2997 $(2,2 \%)$, mostrando um subregistro relacionado a esse item. Nota-se uma porcentagem significativa de $24,5 \%$ de mães entre 10 a 19 anos, correspondendo a 32937 casos.

Quanto à escolaridade das mães, esta variou de analfabetismo até ensino superior completo. A escolaridade da $5^{\circ}$ a $8^{\circ}$ série incompleta foi a mais observada (32229/24\%). As analfabetas representaram um pequeno percentual de 0,8\% (1059 casos). Apenas 1014 (0,7\%) mulheres possuíam ensino superior completo. Também foi observado um subregistro nesta variável, com $37916(28,2 \%)$ casos com faixa etária ignorada.

No que se refere à realização de pré-natal das gestantes das quais os recémnascidos tiveram diagnóstico de sífilis congênita, observou-se que a grande maioria (106468/79,2\%) realizou o pré-natal durante o período estudado. A frequência de realização de pré-natal mostrou-se ascendente ao longo dos anos. A quantidade de pré-natais não realizado se mostra maior que o valor dos ignorados em todos os anos, sendo os ignorados com um número de 7573 (5,6\%) casos, mostrando que esta variável tem um subregistro menor, quando comparada à escolaridade das mães.

A respeito do momento de diagnóstico de sífilis materna, a maioria foi realizado durante o pré-natal $(71114 / 52,9 \%)$, porém, também houveram porcentagens expressivas de diagnóstico no momento do parto ou curetagem (45858/34,1\%) e após o parto (10696/ 7,9\%).

Sobre as gestantes que realizaram tratamento para sífilis, houve predominância do tratamento inadequado, que representou 56,7\% (76229) dos casos, com o percentual máximo de $58,7 \%$ em 2016, mostrando que há falhas assistência à saúde das gestantes. O tratamento adequado representou apenas $4,2 \%$ (5638) dos casos. É preocupante o fato de que $27,2 \%$ (36582) das mães não 
realizaram o tratamento para sífilis, e que a variável foi ignorada em 11,8\% (15960) dos casos, havendo certo grau de subregistro.

No que se diz respeito ao tratamento para sífilis pelo parceiro da mãe, apenas $15,6 \%$ (20944) receberam o tratamento, por outro lado a não realização do tratamento representa mais da metade dos casos (81735/ 60,8\%). A variável também teve um valor considerável de subregistro, com um percentual de $23,6 \%$ (31730) de ignorados. A quantidade de parceiros tratados aumentou progressivamente ao longo de cada ano, com valor máximo 5838 (22,2\%) em 2018, porém em nenhum ano ultrapassou o número de não tratados, que sempre se manteve acima do dos $50 \%$ em todos os anos investigados.

Tabela 2 - Casos de sífilis congênita segundo realização de pré-natal, faixa etária e escolaridade da mãe, tratamento da mãe e do parceiro da mãe, por ano de diagnóstico. Brasil, 2012-2018.

\begin{tabular}{|c|c|c|c|c|c|c|c|c|}
\hline Brasil & 2012 & 2013 & 2014 & 2015 & 2016 & 2017 & 2018 & Total \\
\hline & $n(\%)$ & $n(\%)$ & $n(\%)$ & $n(\%)$ & $\mathrm{n}(\%)$ & $n(\%)$ & $n(\%)$ & $n(\%)$ \\
\hline \multicolumn{9}{|c|}{ Realização de pré-natal } \\
\hline Sim & $\begin{array}{c}8558 \\
(73,3)\end{array}$ & $\begin{array}{l}10482 \\
(74,8)\end{array}$ & $\begin{array}{l}12667 \\
(77,5)\end{array}$ & $\begin{array}{l}15484 \\
(78,5)\end{array}$ & $\begin{array}{l}17323 \\
(81,2)\end{array}$ & $\begin{array}{l}20438 \\
(81,7)\end{array}$ & $\begin{array}{l}21516 \\
(81,8)\end{array}$ & $\begin{array}{c}106468 \\
(79,2)\end{array}$ \\
\hline Não & $\begin{array}{l}2411 \\
(20,6)\end{array}$ & $\begin{array}{c}2598 \\
(18,5)\end{array}$ & $\begin{array}{c}2745 \\
(16,8)\end{array}$ & $\begin{array}{c}2933 \\
(14,9)\end{array}$ & $\begin{array}{l}2885 \\
(13,5)\end{array}$ & $\begin{array}{c}3284 \\
(13,1)\end{array}$ & $\begin{array}{c}3512 \\
(13,3)\end{array}$ & $\begin{array}{l}20368 \\
(15,1)\end{array}$ \\
\hline Ignorado & $710(6,1)$ & $929(6,6)$ & $942(5,8)$ & $1302(6,6)$ & $1123(5,3)$ & $1287(5,1)$ & $1280(4,9)$ & $7573(5,6)$ \\
\hline Total & $\begin{array}{l}11679 \\
(100)\end{array}$ & $\begin{array}{l}14009 \\
(100)\end{array}$ & $\begin{array}{l}16354 \\
(100)\end{array}$ & $\begin{array}{l}19719 \\
(100)\end{array}$ & $\begin{array}{c}21331 \\
(100)\end{array}$ & $\begin{array}{c}25009 \\
(100)\end{array}$ & $\begin{array}{c}26308 \\
(100)\end{array}$ & $\begin{array}{c}134.409 \\
(100)\end{array}$ \\
\hline \multicolumn{9}{|c|}{ Momento de diagnóstico de sífilis materna } \\
\hline $\begin{array}{l}\text { No pré- } \\
\text { natal }\end{array}$ & $\begin{array}{c}4944 \\
(42,3)\end{array}$ & $\begin{array}{c}6289 \\
(44,9)\end{array}$ & $\begin{array}{c}7911 \\
(48,4)\end{array}$ & $\begin{array}{l}10153 \\
(51,5)\end{array}$ & $\begin{array}{l}12260 \\
(57,5)\end{array}$ & $\begin{array}{l}14413 \\
(57,6)\end{array}$ & $\begin{array}{l}15144 \\
(57,6)\end{array}$ & $\begin{array}{l}71114 \\
(52,9)\end{array}$ \\
\hline $\begin{array}{c}\text { No } \\
\text { momento } \\
\text { do parto } \\
\text { /curetagem }\end{array}$ & $\begin{array}{l}4834 \\
(41,4)\end{array}$ & $\begin{array}{c}5439 \\
(38,8)\end{array}$ & $\begin{array}{l}5966 \\
(36,5)\end{array}$ & $\begin{array}{c}6838 \\
(34,7)\end{array}$ & $\begin{array}{c}6582 \\
(30,9)\end{array}$ & $\begin{array}{l}7845 \\
(31,4)\end{array}$ & $8354(31,8)$ & $\begin{array}{l}45858 \\
(34,1)\end{array}$ \\
\hline $\begin{array}{l}\text { Após o } \\
\text { parto }\end{array}$ & $\begin{array}{c}1275 \\
(10,9)\end{array}$ & $\begin{array}{c}1478 \\
(10,6)\end{array}$ & $\begin{array}{c}1664 \\
(10,2)\end{array}$ & $1614(8,2)$ & $1560(7,3)$ & $1634(6,5)$ & $1471(5,6)$ & $\begin{array}{c}10696 \\
(7,9)\end{array}$ \\
\hline $\begin{array}{l}\text { Não } \\
\text { realizado }\end{array}$ & $61(0,5)$ & $98(0,7)$ & $107(0,7)$ & $120(0,6)$ & $108(0,5)$ & $151(0,6)$ & $198(0,8)$ & $843(0,6)$ \\
\hline Curetagem & $565(4,8)$ & $705(5,0)$ & $706(4,3)$ & $994(5,0)$ & $821(3,8)$ & $966(3,9)$ & $1141(4,3)$ & $5898(4,4)$ \\
\hline Total & $\begin{array}{l}11679 \\
(100)\end{array}$ & $\begin{array}{l}14009 \\
(100)\end{array}$ & $\begin{array}{l}16354 \\
(100)\end{array}$ & $\begin{array}{l}19719 \\
(100)\end{array}$ & $\begin{array}{l}21331 \\
(100)\end{array}$ & $\begin{array}{c}25009 \\
(100)\end{array}$ & $\begin{array}{c}26308 \\
(100)\end{array}$ & $\begin{array}{c}134409 \\
(100)\end{array}$ \\
\hline \multicolumn{9}{|c|}{ Faixa etária da mãe } \\
\hline 10 a 14 & $153(1,3)$ & $150(1,1)$ & $152(0,9)$ & $190(1,0)$ & $221(1,0)$ & $222(0,9)$ & $231(0,9)$ & $1319(1)$ \\
\hline
\end{tabular}




\begin{tabular}{|c|c|c|c|c|c|c|c|c|}
\hline 15 a 19 & $\begin{array}{c}2598 \\
(22,2)\end{array}$ & $\begin{array}{c}3328 \\
(23,8)\end{array}$ & $\begin{array}{c}3843 \\
(23,5)\end{array}$ & $\begin{array}{c}4675 \\
(23,7)\end{array}$ & $\begin{array}{c}5012 \\
(23,5)\end{array}$ & $\begin{array}{c}6011 \\
(24,0)\end{array}$ & $\begin{array}{c}6151 \\
(23,4)\end{array}$ & $\begin{array}{l}31618 \\
(23,5)\end{array}$ \\
\hline 20 a 29 & $\begin{array}{c}6017 \\
(51,5)\end{array}$ & $\begin{array}{c}7041 \\
(50,3)\end{array}$ & $\begin{array}{c}8461 \\
(51,7)\end{array}$ & $\begin{array}{l}10205 \\
(51,8)\end{array}$ & $\begin{array}{l}11284 \\
(52,9)\end{array}$ & $\begin{array}{l}13372 \\
(53,5)\end{array}$ & $\begin{array}{l}14109 \\
(53,6)\end{array}$ & $\begin{array}{l}70489 \\
(52,4)\end{array}$ \\
\hline 30 a 39 & $\begin{array}{c}2387 \\
(20,4)\end{array}$ & $\begin{array}{c}2787 \\
(19,9)\end{array}$ & $\begin{array}{c}3202 \\
(19,6)\end{array}$ & $\begin{array}{c}3720 \\
(18,9)\end{array}$ & $\begin{array}{c}3878 \\
(18,2)\end{array}$ & $\begin{array}{c}4411 \\
(17,6)\end{array}$ & $\begin{array}{c}4726 \\
(18,0)\end{array}$ & $\begin{array}{l}25111 \\
(18,7)\end{array}$ \\
\hline 40 ou mais & $250(2,1)$ & $292(2,1)$ & $309(1,9)$ & $387(2,0)$ & $418(2,0)$ & $461(1,8)$ & $484(1,8)$ & $2601(1,9)$ \\
\hline Ignorado & $274(2,3)$ & $411(2,9)$ & $387(2,4)$ & $542(2,7)$ & $518(2,4)$ & $532(2,1)$ & $607(2,3)$ & $2997(2,2)$ \\
\hline Total & $\begin{array}{l}11679 \\
(100)\end{array}$ & $\begin{array}{l}14009 \\
(100)\end{array}$ & $\begin{array}{l}16354 \\
(100)\end{array}$ & $\begin{array}{l}19719 \\
(100)\end{array}$ & $\begin{array}{l}21331 \\
(100)\end{array}$ & $\begin{array}{l}25009 \\
(100)\end{array}$ & $\begin{array}{c}26308 \\
(100)\end{array}$ & $\begin{array}{c}134409 \\
(100)\end{array}$ \\
\hline \multicolumn{9}{|c|}{ Escolaridade da mãe } \\
\hline Analfabeto & $163(1,4)$ & $161(1,1)$ & $155(0,9)$ & $160(0,8)$ & $145(0,7)$ & $131(0,5)$ & $144(0,5)$ & $1059(0,8)$ \\
\hline $\begin{array}{l}1^{\circ} \text { a } 4^{\circ} \\
\text { série inc. }\end{array}$ & $1.037(8,9)$ & $1040(7,4)$ & $1147(7,0)$ & $1077(5,5)$ & $997(4,7)$ & $1158(4,6)$ & $1102(4,2)$ & $7558(5,6)$ \\
\hline $\begin{array}{l}4^{\circ} \text { série } \\
\text { comp. }\end{array}$ & $748(6,4)$ & $698(5,0)$ & $675(4,1)$ & $749(3,8)$ & $714(3,3)$ & $753(3,0)$ & $738(2,8)$ & $5075(3,7)$ \\
\hline $\begin{array}{c}5^{\circ} \text { a } 8^{\circ} \\
\text { série inc. }\end{array}$ & $\begin{array}{c}2933 \\
(25,1)\end{array}$ & $\begin{array}{c}3466 \\
(24,7)\end{array}$ & $\begin{array}{c}4138 \\
(25,3)\end{array}$ & $\begin{array}{c}4844 \\
(24,6)\end{array}$ & $\begin{array}{l}5220 \\
(24,5)\end{array}$ & $\begin{array}{c}5792 \\
(23,2)\end{array}$ & $\begin{array}{c}5836 \\
(22,2)\end{array}$ & $\begin{array}{l}32229 \\
(24,0)\end{array}$ \\
\hline $\begin{array}{l}\text { Fund. } \\
\text { Comp. }\end{array}$ & $966(8,3)$ & $1166(8,3)$ & $1337(8,2)$ & $\begin{array}{c}2101 \\
(10,7)\end{array}$ & $\begin{array}{c}2133 \\
(10,0)\end{array}$ & $\begin{array}{c}2777 \\
(11,1)\end{array}$ & $\begin{array}{c}2830 \\
(10,8)\end{array}$ & $\begin{array}{c}13310 \\
(9,9)\end{array}$ \\
\hline Mé & $993(8,5)$ & $1284(9,2)$ & $\begin{array}{c}1750 \\
(10,7)\end{array}$ & $\begin{array}{l}2134 \\
(10,8)\end{array}$ & $\begin{array}{l}2547 \\
(11,9)\end{array}$ & $\begin{array}{c}3362 \\
(13,4)\end{array}$ & $\begin{array}{c}3429 \\
(13,0)\end{array}$ & $\begin{array}{l}15499 \\
(11,5)\end{array}$ \\
\hline $\begin{array}{l}\text { Méd. } \\
\text { Comp. }\end{array}$ & $\begin{array}{c}1171 \\
(10,0)\end{array}$ & $\begin{array}{c}1550 \\
(11,1)\end{array}$ & $\begin{array}{c}2002 \\
(12,2)\end{array}$ & $\begin{array}{c}2581 \\
(13,1)\end{array}$ & $\begin{array}{c}3151 \\
(14,8)\end{array}$ & $\begin{array}{c}3957 \\
(15,8)\end{array}$ & $\begin{array}{c}4487 \\
(17,1)\end{array}$ & $\begin{array}{l}18899 \\
(14,1)\end{array}$ \\
\hline Sup. Inc. & $66(0,6)$ & $92(0,7)$ & $120(0,7)$ & $156(0,8)$ & $200(0,9)$ & $259(1,0)$ & $292(1,1)$ & $1185(0,9)$ \\
\hline $\begin{array}{l}\text { Sup. } \\
\text { Comp. }\end{array}$ & $76(0,7)$ & $73(0,5)$ & $103(0,6)$ & $131(0,7)$ & $159(0,7)$ & $228(0,9)$ & $244(0,9)$ & $1014(0,7)$ \\
\hline $\begin{array}{l}\text { Não se } \\
\text { aplica }\end{array}$ & $52(0,4)$ & $88(0,6)$ & $93(0,6)$ & $106(0,5)$ & $87(0,4)$ & $124(0,5)$ & $115(0,4)$ & $665(0,5)$ \\
\hline Ignorado & $\begin{array}{c}3474 \\
(29,7)\end{array}$ & $\begin{array}{c}4391 \\
(31,3)\end{array}$ & $\begin{array}{c}4834 \\
(29,6)\end{array}$ & $\begin{array}{c}5680 \\
(28,8)\end{array}$ & $\begin{array}{c}5978 \\
(28,0)\end{array}$ & $\begin{array}{c}6468 \\
(25,9)\end{array}$ & $\begin{array}{l}7091 \\
(27,0)\end{array}$ & $\begin{array}{l}37916 \\
(28,2)\end{array}$ \\
\hline Total & $\begin{array}{l}11679 \\
(100)\end{array}$ & $\begin{array}{l}14009 \\
(100)\end{array}$ & $\begin{array}{l}16354 \\
(100)\end{array}$ & $\begin{array}{l}19719 \\
(100)\end{array}$ & $\begin{array}{l}21331 \\
(100)\end{array}$ & $\begin{array}{c}25009 \\
(100)\end{array}$ & $\begin{array}{c}26308 \\
(100)\end{array}$ & $\begin{array}{c}134409 \\
(100)\end{array}$ \\
\hline \multicolumn{9}{|c|}{ Tratamento da mãe } \\
\hline Adequado & $373(3,2)$ & $425(3,0)$ & $595(3,6)$ & $802(4,1)$ & $853(4,0)$ & $1114(4,5)$ & $1476(5,6)$ & $5638(4,2)$ \\
\hline Inadequado & $\begin{array}{c}6292 \\
(53,9)\end{array}$ & $\begin{array}{c}7978 \\
(56,9)\end{array}$ & $\begin{array}{c}9547 \\
(58,4)\end{array}$ & $\begin{array}{l}11223 \\
(56,9)\end{array}$ & $\begin{array}{l}12521 \\
(58,7)\end{array}$ & $\begin{array}{l}14305 \\
(57,2)\end{array}$ & $\begin{array}{l}14363 \\
(54,6)\end{array}$ & $\begin{array}{l}76229 \\
(56,7)\end{array}$ \\
\hline $\begin{array}{c}\text { Não } \\
\text { Realizado }\end{array}$ & $\begin{array}{c}3712 \\
(31,8)\end{array}$ & $\begin{array}{c}3915 \\
(27,9)\end{array}$ & $\begin{array}{c}4419 \\
(27,0)\end{array}$ & $\begin{array}{c}5363 \\
(27,2)\end{array}$ & $\begin{array}{c}5639 \\
(26,4)\end{array}$ & $\begin{array}{c}6595 \\
(26,4)\end{array}$ & $\begin{array}{c}6939 \\
(26,4)\end{array}$ & $\begin{array}{l}36582 \\
(27,2)\end{array}$ \\
\hline Ignorado & $\begin{array}{c}1302 \\
(11,1)\end{array}$ & $\begin{array}{c}1691 \\
(12,1)\end{array}$ & $\begin{array}{c}1793 \\
(11,0)\end{array}$ & $\begin{array}{c}2331 \\
(11,8)\end{array}$ & $\begin{array}{l}2318 \\
(10,9)\end{array}$ & $\begin{array}{l}2995 \\
(12,0)\end{array}$ & $\begin{array}{c}3530 \\
(13,4)\end{array}$ & $\begin{array}{l}15960 \\
(11,8)\end{array}$ \\
\hline Total & $\begin{array}{l}11679 \\
(100)\end{array}$ & $\begin{array}{l}14009 \\
(100)\end{array}$ & $\begin{array}{l}16354 \\
(100)\end{array}$ & $\begin{array}{l}19719 \\
(100)\end{array}$ & $\begin{array}{l}21331 \\
(100)\end{array}$ & $\begin{array}{c}25009 \\
(100)\end{array}$ & $\begin{array}{c}26308 \\
(100)\end{array}$ & $\begin{array}{c}134.409 \\
(100)\end{array}$ \\
\hline \multicolumn{9}{|c|}{ Tratamento do parceiro da mãe } \\
\hline Sim & $\begin{array}{c}1315 \\
(11,3)\end{array}$ & $\begin{array}{c}1614 \\
(11,5)\end{array}$ & $\begin{array}{c}2169 \\
(13,3)\end{array}$ & $\begin{array}{c}2749 \\
(13,9)\end{array}$ & $\begin{array}{c}3254 \\
(15,3)\end{array}$ & $\begin{array}{c}4005 \\
(16,0)\end{array}$ & $\begin{array}{c}5838 \\
(22,2)\end{array}$ & $\begin{array}{l}20944 \\
(15,6)\end{array}$ \\
\hline Não & $\begin{array}{c}7645 \\
(65,5)\end{array}$ & $\begin{array}{c}8749 \\
(62,5)\end{array}$ & $\begin{array}{l}10315 \\
(63,1)\end{array}$ & $\begin{array}{l}12340 \\
(62,6)\end{array}$ & $\begin{array}{l}13297 \\
(62,3)\end{array}$ & $\begin{array}{l}15383 \\
(61,5)\end{array}$ & $\begin{array}{l}14006 \\
(53,2)\end{array}$ & $\begin{array}{l}81735 \\
(60,8)\end{array}$ \\
\hline Ignorado & $\begin{array}{l}2719 \\
(23,3)\end{array}$ & $\begin{array}{c}3646 \\
(26,0)\end{array}$ & $\begin{array}{c}3870 \\
(23,7)\end{array}$ & $\begin{array}{c}4630 \\
(23,5)\end{array}$ & $\begin{array}{c}4780 \\
(22,4)\end{array}$ & $\begin{array}{c}5621 \\
(22,5)\end{array}$ & $\begin{array}{l}6464 \\
(24,6)\end{array}$ & $\begin{array}{l}31730 \\
(23,6)\end{array}$ \\
\hline
\end{tabular}




\begin{tabular}{|ccccccccc|}
\hline \multirow{2}{*}{ Total } & 11679 & 14009 & 16354 & 19719 & 21331 & 25009 & 26308 & 134.409 \\
& $(100)$ & $(100)$ & $(100)$ & $(100)$ & $(100)$ & $(100)$ & $(100)$ & $(100)$ \\
\hline
\end{tabular}

Fonte: MS/SVS/SINAN - Departamento de Doenças de Condições Crônicas e Infecções Sexualmente Transmissíveis.

\section{DISCUSSÃO}

O aumento de casos de sífilis congênita ao longo do período estudado pode ser atribuído a vários fatores, como diminuição dos casos subregistrados e em consequência o aumento das notificações, bem como maiores esforços do serviço de saúde em identificar e notificar os casos. Houve predominância de realização de prénatal pelas mães com uma porcentagem de $79,2 \%$, porém apenas $52,9 \%$ das gestantes receberam o diagnóstico materna durante o pré-natal, sendo $34,1 \%$ dos diagnósticos realizados no momento do parto ou curetagem e 7,9\% realizados após o parto, esses dados indicam que o serviço de pré-natal não está sendo totalmente eficiente em diagnosticar a sífilis gestacional, e assim iniciar um tratamento para prevenir a sífilis congênita.

Além disso, muitas maternidades não disponibilizam o VDRL como rotina para parturientes, de forma que o bebê e a mãe retornam a comunidade com a chance de possuírem a infecção. Essas falhas levam ao aumento de casos de sífilis congênita, de forma que há a necessidade de ações e políticas públicas adequadas para erradicar a doença, que é fácil de controlar (COSTA et al., 2013).

Desta forma, a existência da sífilis congênita está associada ao acesso e baixa qualidade do pré-natal. É alarmante que muitas mulheres ainda não possuem acesso à serviços de pré- natal. $E$ também há mulheres que realizam consultas prénatais e possuem sorologia positiva para sífilis, porém não voltam para buscar os resultados dos exames, as que não foram tratadas de forma adequada, ou que não tiveram seus parceiros tratados durante sua estação (COSTA et al., 2013).

Em relação à faixa etária, foi observado que a mais presente, sendo mais da metade, foram de mulheres de 20 a 29 anos, seguida de 15 a 19 anos, mostrando que a maioria são adolescentes e adultas jovens. No período, foram notificados 
1319 casos de sífilis congênitas em bebes com mães de 10 a 14 anos. Isso mostra que muitas adolescentes possuem uma vida sexual precoce e desprotegida, o que demonstra a necessidade de orientações sobre sexo seguro e incentivo ao adiamento de iniciação sexual (COSTA et al., 2013).

Também foi observado que em relação à escolaridade, a que prevaleceu foram as mães que estudaram até a $5^{\circ}$ a $8^{\circ}$ série incompleta. Provavelmente, mulheres com um menor nível de instrução possuem um menor acesso à informação para evitar a infecção e a transmissão vertical, e algumas que têm o diagnóstico confirmado não compreendem a importância de realizarem um tratamento adequado (CARVALHO; BRITO, 2014).

Outro dado que chama atenção são os casos de sífilis congênitas em mães com ensino médio completo, superior incompleto e superior completo, porque pressupõe-se que esta população possua alguma informação sobre as Infecções Sexualmente Transmissíveis (IST) e suas prevenção, e sobre a necessidade de fazer um pré-natal (COSTA et al., 2013).

No estudo foi observado que apenas $4,2 \%$ das gestantes receberam um tratamento adequado para sífilis. É preciso enfatizar que a prevenção da infecção tem um valor baixo, porém o tratamento de uma criança sífilis congênita é caro e demorado (COSTA et al., 2013). Quando o recém-nascido tem um diagnóstico positivo de sífilis congênita, ele é internado e tratado com antibiótico, utilizando de maiores recursos (BRITO; KIMURA, 2018).

Por outo lado o tratamento inadequado das mães representou $56,7 \%$ dos casos, e $27,2 \%$ das mães não realizaram tratamento para sífilis. A prevenção da sífilis, seja ela adquirida ou congênita, é conhecida e dispersada nos ambientes de saúde, porém, faz-se necessário técnicas mais apropriadas para implantá-la (SOUZA; BECK, 2019). É necessário a continuação de pesquisas e dedicação humana para diagnosticar, tratar e, por consequência, erradicar a sífilis congênita no mundo. Ainda atingindo a saúde materno-infantil, esta infecção apenas será extinta nas Américas quando houverem serviços pré-natal de elevada qualidade (COOPER et al., 2016).

O fato de $60,8 \%$ dos parceiros das mães não receberem um tratamento adequado eleva o risco de reinfecção da mãe mesmo que esta seja tratada, e 
aumenta a possibilidade de transmissão vertical. Isto mostra que há uma falha na infraestrutura na saúde pública do país, pois o diagnóstico e tratamento é a melhor forma de prevenir a transmissão da infecção na população (COOPER et al., 2016).

A baixa frequência de tratamento dos parceiros sexuais das mães necessita de uma investigação de como os serviços e profissionais da atenção primária à saúde envolvem o homem nos cuidados durante a gestação, se oferecem acolhimento e assistência aos mesmos, desta forma cumprindo o princípio de integralidade (REIS et al, 2018).

É notável a presença de dados ignorados ou em branco, levando a dedução que os profissionais não preenchem os instrumentos de forma correta. A ficha de notificação de sífilis congênita possui orientações acerca de seu preenchimento, que talvez não esteja sendo satisfatório e seja necessário a capacitação dos profissionais para tal fim (COSTA et al., 2013). Os serviços de pré-natal sofrem influencias do contexto de vida dos usuários, entre eles características socioeconômicas. Sua utilização também depende do acesso, sendo dessa forma de primordial importância os serviços de atenção primária (REIS et al, 2018).

Se diagnosticada corretamente, sífilis congênita é uma infecção possível de ser prevenida. A alta abrangência de realização de pré-natal, aliada a valores altos de transmissão vertical demonstram que há dificuldades no serviço para seu controle e intervenção. A possibilidade de transmissão vertical de sífilis aumenta quando há falhas no diagnóstico e tratamento da gestante e de seu parceiro. Desta forma, a orientação adequada de profissionais sobre sífilis congênita, especialmente sobre suas consequências, às gestantes é essencial para diminuir o risco da infecção (SOUZA; BECK, 2019).

\section{CONCLUSÃO}

Apesar de ser uma infecção que apresenta baixo custo e facilidade para sua prevenção, a sífilis congênita permanece como uma infecção de difícil controle no Brasil, e que continua gerando impactos na saúde materna infantil. 
O estudo demonstrou que mesmo com a maioria das mulheres comparecendo às consultas pré-natais, muitas não têm o diagnóstico de sífilis materna confirmado durante esses períodos, e outras quando têm a confirmação, não recebem um tratamento adequado. A maioria dos parceiros das mães também não recebem um tratamento adequado, mostrando como o nosso sistema de saúde não envolve e abrange o homem no pré-natal. Desta maneira, é preciso inserir a figura masculina nos cuidados da gestação.

Os resultados indicam como a assistência pré-natal possui fragilidades e baixa resolubilidade. É necessário que a atenção primária, que é porta de entrada dos serviços de saúde e possui maior vínculo com a comunidade, realize busca ativa, procure por diagnóstico de sífilis na gestante e no seu parceiro, e incentivem à realização de tratamento adequado, prevenindo assim a sífilis congênita.

Para mudar essa realidade, é necessário que o pré-natal do país tenha sua qualidade melhorada. É necessário que os profissionais de saúde passem por capacitações sobre sífilis congênita e sua prevenção, e que estas também englobem o ensino sobre o preenchimento de fixas de notificação da sífilis congênita, pois os dados do SINAN possuem valores que são ignorados e contribuem para o subregistro de diversos aspectos da doença, como escolaridade da mãe e tratamento do parceiro, o que dificulta o conhecimento sobre a real situação da infecção no país. É preciso que os mesmos se empenhem em acolher, identificar e orientar gestantes portadoras de sífilis congênita, e sensibilizá-las para realizarem um tratamento adequado para não fornecer riscos a seu bebê. Desta forma, iremos possuir um pré-natal que seja não só abrangente, mas também eficiente na promoção de saúde e prevenção de doenças como a sífilis congênita. 


\section{REFERÊNCIAS BIBLIOGRÁFICAS}

BRASIL. Ministério da Saúde. Gabinete do Ministro. PORTARIA № 1.459, DE 24 DE JUNHO DE 2011. Institui, no âmbito do Sistema Único de Saúde - SUS - a Rede Cegonha. Diário Oficial República Federativa do Brasil, 27 jun. 2011.

Ministério da Saúde. Gabinete do Ministro. PORTARIA Nº 77, DE 12 DE JANEIRO DE 2012. Dispõe sobre a realização de testes rápidos, na atenção básica, para a detecção de HIV e sífilis, assim como testes rápidos para outros agravos, no âmbito da atenção prénatal para gestantes e suas parcerias sexuais. Diário Oficial República Federativa do Brasil, 12 jan. 2012.

. Ministério da Saúde. Secretaria de Vigilância em Saúde. Departamento de DST, Aids e do Hepatites Virais. Protocolo Clínico e Diretrizes Terapêuticas para Atenção Integral às Pessoas com Infecções Sexualmente Transmissíveis. Brasília: Ministério da Saúde, 2015.

Ministério da Saúde. Secretaria de Vigilância em Saúde. Departamento de Doenças de Condições Crônicas e Infecções Sexualmente Transmissíveis. Protocolo Clínico e Diretrizes Terapêuticas para Atenção Integral às Pessoas com Infecções Sexualmente Transmissíveis (IST). Brasília: Ministério da Saúde, 2019.

BRITO A. P. A.; KIMURA. A. F. Transmissão vertical da sífilis: vivência materna durante a hospitalização para diagnóstico e tratamento de seu filho recém-nascido. Revista Paulista de Enfermagem, v. 29, p. 68-76, 2018.

CARVALHO I. S.; BRITO, R. S. Sífilis congênita no Rio Grande do Norte: estudo descritivo do período 2007-2010, Revista Epidemiologia e Serviços de Saúde, Brasília, v. 23, n. 2. p. $287-$ 294, 2014.

COOPER, J. M. et. al. Em tempo: a persistência da sífilis congênita no Brasil - Mais avanços são necessários! Revista Paulista de Pediatria, São Paulo, v. 34, n. 3, p. 251-253, set. 2016.

COSTA, C. C. et al. Sífilis congênita no Ceará: análise epidemiológica de uma década. Revista da Escola de Enfermagem da USP, v. 47, n. 1, p. 152-9, 2013.

DOMINGUES, R. M. S. M.; LEAL, M. C. Incidência de sífilis congênita e fatores associados à transmissão vertical da sífilis: dados do estudo Nascer no Brasil. Cadernos de Saúde Pública, Rio de Janeiro, v. 32, n. 6, p. 1-12, jun. 2016.

REIS, G. J. et al. Diferenciais intraurbanos da sífilis congênita: análise preditiva por bairros do Município do Rio de Janeiro, Brasil. Cadernos de Saúde Pública, v. 34, n. 9, 2018.

SOUZA, M. H. T.; E. Q. BECK. Compreendendo a sífilis congênita a partir do olhar materno. Revista de Enfermagem da UFSM, Santa Maria, v. 9, ed. 56, p. 1-13, 2019. 